\title{
Control systems of bionic limbs of the new generation
}

\begin{abstract}
The LLC Bionic Natali Company is a startup and has been engaging in creation of bionic artificial limbs of hands for more than 2 years, now company also try to cover topics of leg. From the first steps, the project had been directed on the solution of a problem of development of the domestic bionic functional artificial limb of the hand based on neural network and others algorithms. In the project it had been created the functional system of management, system of tactile feedback which has increased controllability of a functional artificial limb is already realized and integrated, and also the functional bionic artificial limb of the hand or leg. Based on this work it had been done the general representations and practical application of machine training, neural network and others algorithms. The technology of recognition of gestures of electromyography activity based on neural network or an analog of network is the cornerstone. The bracelet is put on a hand (in case of disabled people, a stump), further noninvasive electrodes remove potential difference of neuromuscular activity; by means of an electric circuit there is data handling and their transmission to the processor where by means of a neural network there is a recognition of a gripper or movement of a knee, further data are transferred for control of a bionic hand or leg.
\end{abstract}

Keywords: The bionic artificial limb, neuronal net, electromyography signals, system of control, EMG, bionic natali, recognition the electromyography signals, artificial intelligence, machine learning
Volume 3 Issue 3 - 2017

\author{
Ivaniuk Natallia, Ponimash Zahar, Karimov \\ Vladimir \\ LLC Bionic Natali, Russia
}

Correspondence: Ivaniuk Natallia, LLC Bionic Natali, Russia, Tel+79262625/ I3, Email ivaniuk@bi-on.ru

Received: October 12, 2017 | Published: November 06, 2017

\section{Introduction}

Now, in most cases in the absence of hand artificial limbs, which bear only the cosmetic purposes that, actually, is a manual model without functionality. Electromechanical artificial limbs which allow replacing partially functionality of a full-fledged hand are widespread poorly at present and have limited functionality, and also are available to still limited circle of users. Especially this situation is characteristic of Russia that is explained by influence of several factors: the certain structure of schemes of social insurance causing an order of financing of prosthetics for the state account low consumer ability of the people who have lost a brush and, of course, absence in the market of decisions in various price segments with the significant level of functionality. The technology of recognition of gestures of electromyography activity based on neural network or an analog of network is the cornerstone. The bracelet is put on a hand (in case of disabled people, a stump), further noninvasive electrodes remove potential difference of neuromuscular activity; by means of an electric circuit there is data handling and their transmission to the processor where by means of a neural network there is a recognition of a gripper or movement of a knee, further data are transferred for control of a bionic hand or leg. This article represents the last results of research on creation the system of the control artificial bionic hands or leg by company LLC Bionic Natali.

\section{Results of research}

The goal of research was selection of an algorithm of data processing of electromyography activity of muscles for the best recognition of gripper and also experimental confirmation of developments, carried out within works. Methods which were used in the course of selection of an algorithm-experimental "trial and error method". It is important to note that at the first stages of the project it was planned to select algorithms, processing collected these electromyograms of muscular activity of disabled people from open sources, but as it has become clear later in open access to information practically wasn't, and what managed to be found, was very far from reality and is poorly described regarding process of removal of data that doesn't exclude existence of a mistake in data. As a result the decision to create own experimental database that has been successfully realized has been made. Within the carried-out scientific research works more than 1 year, basic divergences with data of muscular activity were found in disabled people and given to muscular activity at people without ablation. Distinctive feature is the range of frequencies at which the signal registers, and importance of maintenance of a tone of muscular activity at disabled people, otherwise there are artifacts of the movement, which at people without ablations are not registered. For this task now with Ilizarov's center the system of rehabilitation is studied and also the separate product "an electromyography bracelet" which is taken out in the separate company LLC Bi-oN EMG has been created. Working with data, which is obtained from muscular activity, it has been shown that for optimum work of algorithms on capture recognition, it is necessary to execute the following main stages of processing:

a. Filtration.

b. Preprocessing.

c. Then there is already a submission of data on neural network or an algorithm similar to it.

d. Post-data processing, additional training of neural or similar network.

When we tested algorithms, two main variants were developed; the first used simple pre-processing of the signal. And the second neural network hash function as additional preprocessing (Figure 1). 


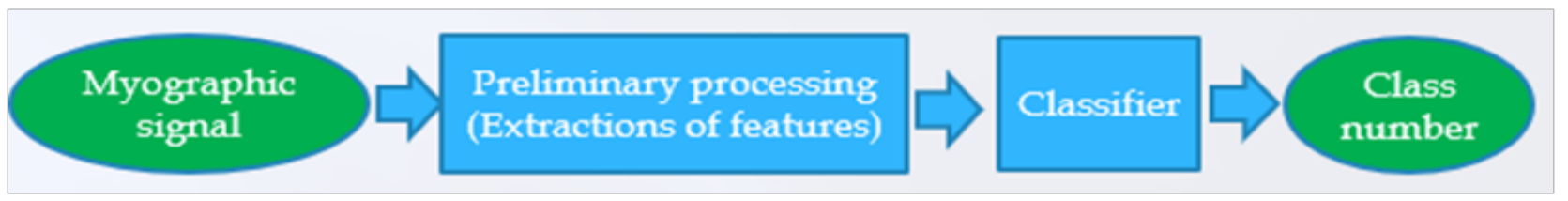

Figure I Without a neural network hash function, the results.

All gestures were recognized with a probability greater than $70 \%$, of these, two gestures were recognized with a probability of less than $80 \%, 6$ with recognized with probability $<90 \%$, but $>80 \%, 8$ gestures $>90 \%$ and 2 with the probability of a business to $100 \%$. This method was tested for real-time amputations. Its accuracy averaged about $90 \%$ (Figure 2). In the first stage (Figure 3), a features space is generated from the signal. After that, a hash function is used based on neural networks. After, the code classifier with the hash function recognizes gestures. But in this test the signals were recorded beforehand. Real-time mode was simulated. This algorithm was tested on various amputees; the algorithm gives a high accuracy of determining the gesture. Below is the test of the algorithm on a person with shoulder amputation, 7 gestures were recognized. The Figure 4 shows the results of testing on 7 gestures. And Figure 5 shows the results of testing on 11 gestures.

\section{Recognition precision. Class № 1: 95,69 \\ Recognition precision. Class № 2: 81,296 \\ Recognition precision. Class № 3: 96,996 \\ Recognition precision. Class № 4: 89,2\% \\ Recognition precision. Class № 5: 79,3\% \\ Recognition precision. Class № 6: 85,9\% \\ Recognition precision. Class № 7: 68,89 \\ Recognition precision. Class № 8: 73,796 \\ Recognition precision. Class № 9: 79,7\% \\ Recognition precision. Class № 10: 80,796 \\ Recognition precision. Class № 11: 85,996 \\ Recognition precision. Class № 12: 83,5\% \\ Recognition precision. Class № 13: 64,3\% \\ Recognition precision. Class № 14: 93,696 \\ Recognition precision. Class № 15: 95,296}

Figure 2 With results of Test.

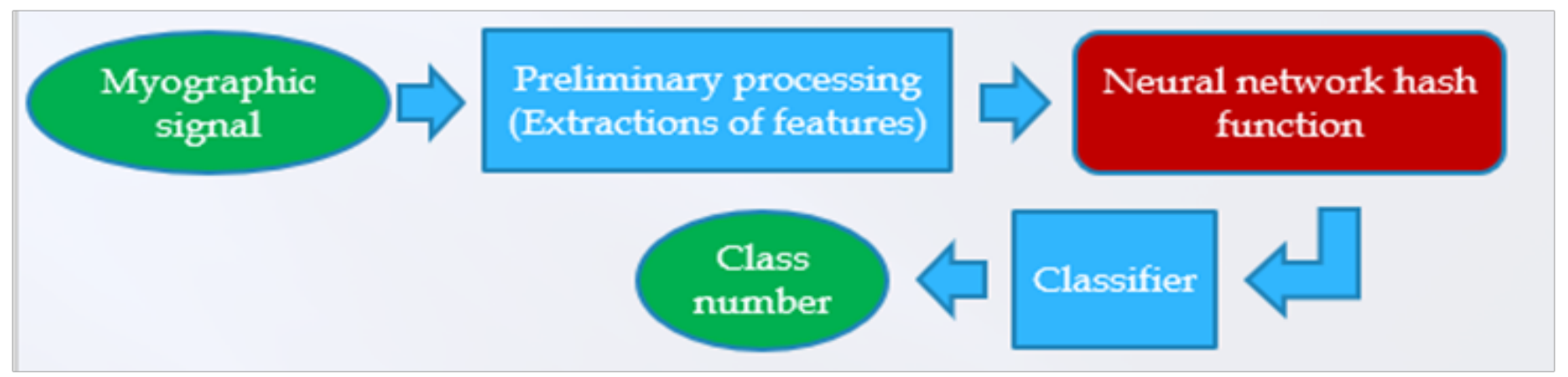

Figure 3 The slide shows the algorithm for recognizing gestures with neural network hash function.

Neural network hash function consists not from one algorithm of neural net; it is complex of transformations, exactly 5 levels of different algorithms. On the entrance on the example there are 8 EMG signals, what had been modified with different neural networks and number of neurons. The details can't be open because it is trade secret of the company LLC Bionic Natali and on this base had been created unique product of recognition of EMG signals of the hand. According to the previous researches described in a source of information ${ }^{1}$ for a condition of implementation of requirements of work in real time, the time of recognition of a signal has to occupy no more than $250 \mathrm{~ms}$. For comfortable work of the user productivity or recognition accuracy (percentage of right cases of classification to all considered cases) has to be not lower than $95 \%$, as shown in a source. ${ }^{2}$ Most details about recognition can be found in a source of information. ${ }^{3}$ 
Recognition precision. Class № 1: 99,66666666666667\%

Recognition precision. Class № 2: $99 \%$

Recognition precision. Class № 3: 98,33333333333333\%

Recognition precision. Class № 4: 99,33333333333333\%

Recognition precision. Class № 5: 98,333333333333333\%

Recognition precision. Class № 6: $99 \%$

Recognition precision. Class № $7: 100 \%$

Figure 4 The results of testing on 7 gestures.

Recognition precision. Class № 1: 98,33333333333333\%

Recognition precision. Class № 2: $99 \%$

Recognition precision. Class № 3: $100 \%$

Recognition precision. Class № 4: 99,66666666666667\%

Recognition precision. Class № 5: $98 \%$

Recognition precision. Class № 6: $98,66666666666667 \%$

Recognition precision. Class № 7: $99 \%$

Recognition precision. Class № 8: 97,333333333333333\%

Recognition precision. Class № 9: 98,3333333333333333\%

Recognition precision. Class № 10: 95,333333333333333\%

Recognition precision. Class № 11: 99,66666666666667\%

Figure 5 The results of testing on II gestures.

\section{Conclusion}

In spite of the fact, that huge amount of works has been done; there is still a big field for activity regarding selection and improvement of an algorithm on recognition of gripper. The similar work can be compared to art as we will com-pare selection of an algorithm to creativity. The current results of the LLC Bionic Natali company in this sphere - it is recognition with probability of $98 \%$ on 14 grippers on 8 sensors with amplifiers from a forearm. The concerning removal of data and recognition of capture in disabled people then in practice were difficulties at movements of muscles and pain at a spasm in long muscular tension. In this regard, there was been made a decision together with many medical centers to develop a method of restoration of muscles and to create the tool for their training. An important component is the mathematical analysis of these artifacts and their elimination for a possibility of practical application of bionic artificial limbs based on neural network and other algorithms in practice. The same methods of control had been analysed for movement of a knee, the first results just showed amazing implementation, muscular activity of stump of leg can be used for control of movements of the knee. The control of sole doesn't need such instruments, because people usually use running artificial limbs for legs.

\section{Acknowledgments}

My research project was partially or fully sponsored by (Innovation Center of Skolkovo and personal funding of LLC Avenir) with grant number (Minigrant №MG67/16 from 20.09.2016). In case of no financial assistance for the research work, provide the information regarding the sponsor.

\section{Conflict of interest}

No conflict of Interest.

\section{References}

1. MSh Haziakhmetov. Properties of window dispersion of a myogramma as casual process. Systems and means inform. 2014;24(3):110-120.

2. Englehart K, Hudgins B. A robust, real-time control scheme for multifunction myoe-lectric control. IEEE Trans Biomed Eng. 2003;50(7):848-854.

3. Ivaniuk N, Ponimash Z, Karimov V. Art of recognition the electromyographic signals for control of the bionic artificial limb of the hand. International Conference of Artificial Intelligence, Medical Engineering, Education, Advances in Artificial Systems for Medicine and Education. 2017;658:176-181. 